\title{
Antioxidant (Antiradical) Compounds
}

\author{
Monica Butnariu ${ }^{1 *}$ and loana Grozea ${ }^{2}$ \\ ${ }^{1}$ Chemistry and Vegetal Biochemistry, Romania \\ ${ }^{2}$ Plant Protection, Banat's University of Agricultural Sciences and Veterinary Medicine from Timisoara, Romania
}

Abbreviations: FR: Free Radicals; AA: Antioxidants; TCP: Tocopherols; BHA: Buthylhydroxyanisol; BHT: butylhydroxytoluene; THBP: Trihydroxybutyrophenon; TBHQ: Tert-Butyl-Hydroquinone; SOD: Superoxide Dismutase; CAT: Catalase; GRx: Glutathione Peroxidase; DNA: Deoxyribonucleic Acid; GST: Glutathione-STransferase; HRP: Horseradish Peroxidase; ROS: Radical Oxygen Species

Antioxidants (AA) slow down the process of degradation so that the energetic action of the environment can lead to higher sustainability. They interact with FR, making possible their reaction with oxygen. Antioxidants can be grouped into two classes-synthesis antioxidants and natural antioxidants. The difference between the two categories is that most synthesis antioxidants generate substances that develop cancer or other diseases [1]. Classifying antioxidants can be done depending on their function or on their nature. Depending on their function, there are:

- Primary antioxidants (antioxidants proper): ascorbic acid and its derivatives, tocopherols, the esters of gallic acid, erythorbic acid and its sodium salt, BHA, BHT and other substances THBP and TBHQ.

- Secondary antioxidants (substances with antioxidant action but that have other functions as well). Sulphur dioxide and sulphites as well as lecithin are secondary antioxidants.

Besides the above-mentioned compounds, there are also substances that can strengthen the action of primary antioxidants. Such compounds as lactic acid and lactates, citric acid and citrates, tartric acid and tartrates, etc. have either a synergetic or action of metal complexes that catalysis self-oxidation reactions [2]. Antioxidants can be: AA for lipid systems; and AA for hydrophilic systems.

Depending on their nature, AA can be natural or synthesis. Tocopherols (TCP) are natural AA, as well as a series of compounds with different structures that can be found in plants (flavones). The most important class of synthesis AA act by interrupting the lipid selfoxidation reaction chain.

The phenols substituted belong to this category-thy react with peroxidic FR and release hydrogen from the $\mathrm{OH}$ phenolic group:

\section{$\mathrm{ROO}^{\circ}+\mathrm{Ar}-\mathrm{OH} \rightarrow \mathrm{ROOH}+\mathrm{Ar}-\mathrm{O}^{\circ}$.}

The FR of the antioxidant is stabilised due to the structure of the molecule, the $\mathrm{OH}$ group of the antioxidant neighbouring, in general, one or several voluminous groups (tert-butyl). Another possibility of stabilising the radical is through meso-isomerism. These radicals can react with peroxidic radicals or between them, forming compounds that no longer have a radicalic character:

$\mathrm{ROO}^{\circ}+\mathrm{AH} \rightarrow \mathrm{ROOH}+\mathrm{A}^{\circ}$ and $\mathrm{ROO}^{\circ}+\mathrm{A}^{\circ} \rightarrow \mathrm{ROOA}$.

After the antioxidant is exhausted in these reactions, the process of self-oxidation starts again, at high speed. To prolong the duration of action of the antioxidants, we can use reducing agents $(-\mathrm{C}-\mathrm{SH}$ or $\mathrm{R}-$ $S H)$, capable of regenerating the antioxidant by releasing a hydrogen.
The use of two AA can have a favourable effect due to their synergism, their optimal ratio being established for each case apart. AA is efficient in a single domain of concentration: at higher concentrations, it can have even a pro-oxidative effect. Another possible mechanism through which antioxidants act is oxygen capture, which no longer gets to oxidate the lipid substratum [3]. A third possible mechanism of action of antioxidants is the inhibition of some oxidative enzymes such as polyphenol oxidase or lipoxygenase, which prevent oxidation reactions catalysed by these enzymes.

No matter the action place and the nature of the substratum, all AA molecules can be classified by comparing their action mechanisms: the first group contains molecules that have enzymatic action, i.e. AA enzymes. From a mechanistic point of view, their action involves a change of the substratum $\left(\mathrm{O}^{2-}\right.$, which is turned into $\mathrm{H}_{2} \mathrm{O}_{2}$ through SOD). Enzymes are spread in the cells and act together (this is the case of SOD, CAT) catalytically, which means that they are generated continuously. The other molecules can be included in the second group, non-enzymatic AA molecules.

Upon interaction with the radicals, these substances form radical products that can be stable and persisting [4]. These reactions are, usually, stoichiometric, i.e. the molecule of the AA is consumed during the reaction and it needs to be regenerated from other molecules or from other reactions. These substances can be found in different cell compartments, depending on their polarity: vitamins A and $\mathrm{E}$ are in hydrophobic areas, while vitamin C and GST are in hydrophilic areas. Despite their location in different parts inside the cells, hydrophobic and hydrophilic molecules act together.

The tocopheryl radical is generated by ascorbic acid with formation of ascorbil radical, which, in its turn, is reduced by an electron donor compound. AA can be grouped into three categories:

- Preventive antioxidants, that reduce the formation of radicals and of ROS by decomposing hydrogen peroxide and hydroperoxide without generating FR (GPx, GST, HRP, CAT) by capturing metal ions (apoferritin, transferrin, lactoferrin, ceruloplasmin) and by deactivating active oxygen (carotenoids, SOD).

- Radical-deactivating antioxidants, that inhibit the initiation stage and interrupt the propagation stage by capturing radicals

${ }^{*}$ Corresponding author: Monica Butnariu, Chemistry and Vegetal Biochemistry, 300645, Calea Aradului 119, Timis, Romania, Tel: +40-0-256-277-464; Fax: +40-0 256-200-296; E-mail: monicabutnariu@yahoo.com / monica_butnariu@usab-tm.ro

Received July 13, 2012; Accepted July 16, 2012; Published July 18, 2012

Citation: Butnariu M, Grozea I (2012) Antioxidant (Antiradical) Compounds. J Bioequiv Availab 4: xvii-xix. doi:10.4172/jbb.10000e18

Copyright: (c) 2012 Butnariu M, et al. This is an open-access article distributed under the terms of the Creative Commons Attribution License, which permits unrestricted use, distribution, and reproduction in any medium, provided the original author and source are credited. 
before they reach target-cells (vitamin C, uric acid, albumin, bilirubin, vitamin E, carotenoids).

- Molecules involved in recovery and in de novo mechanisms (phosphorlipases, proteases, transferases).

Natural AA can be found in all plant parts and they include carotenoids, vitamins, phenols, and flavonoids. The antioxidants categories and their functions are the following: enzymes and cofactors; metal-bonding molecules (ferritin, transferrin and albumin); mineral salts vitamins (vitamins A, C and E); pigments (carotenoids, polyphenols such as flavonoids, antocyanins, tannins, and chlorophyll).

Some natural or synthesis AA are also used in the food industry to prevent a reduction of fat oxidation (or of other components of foods) during the period of preservation (food additives) [5]. Antioxidant substances are hard to synthesise and they have a high reduction potential, i.e. the possibility of releasing $\mathrm{InH}$. The process of inhibition is as follows:

\section{$\mathrm{InH}+\mathrm{RO}_{2} \rightarrow \mathrm{RO}_{2} \mathrm{H}+\mathrm{In}$ or $\mathrm{InH}+\mathrm{R} \rightarrow \mathrm{RH}+\mathrm{In}$.}

In is an inactive or low active radical. In all cases, together with the increase of the duration of inactivation, there is decrease of the amount of AA; the increase of the peroxide index occurs only after the important decrease of the added AA.

Between the structure of the AA and their efficacy, there is a correlation determined by the following factors: the AA effect increases proportionally with the length of the chain (alkylation's in the meta position is less efficient); the esterification of the hydroxyl groups causes a complete disappearance of the antioxidant activity; the introduction of the allylic groups in the ortho or para position of hydroxyl groups has a favourable effect and the introduction, in the aromatic nucleus of the phenol or of the naphtol, of a secondary or tertiary hydroxyl group increases efficiency.

There is a range of AA that can be sued in the stabilisation of food products, but their use is limited by sanitary regulations. AA protection represents the defense mechanism of the body against oxidation. An AA is a molecule which, compared to the oxidable substance/material is present in low concentrations and slow down or completely stop its oxidation [6]. The resistance of antioxidant effect can be characterized by the so-called induction time. The more efficient the AA, the longer the induction time and the more delayed the oxidation of the molecule attacked [7].

Depending on the action mechanism, AA can be primary or secondary antioxidants.

Primary antioxidants, also called chain-breaking antioxidants, are compounds capable of neutralizing lipid FR, mainly by stopping their radical state by hydrogen transfer and, periodically, by low reactivity compounds. These relatively stable compounds cannot participate in the propagation of the lipid per oxidation, hence the breaking of the chain reaction. Primary AA is effective during the induction period; in their presence, oxidation starts later. Primary AA is formed of molecules of phenol, TPC, gallic acid and its derivatives, flavonoids, and other compounds.

Secondary (preventive) AA prevent lipid oxidation in a different way, inhibiting the initiation by the fact that they oxidate themselves instead to oxidize lipid molecules or they turn intermediary or final products generated during the reactions into non-toxic forms [8].
Phospholipids that synergise with primary AA or with citric acid are included in the category of natural AA. Antioxidants are characterised by the fact that most of them can prevent/inhibit through several types of action mechanisms resulting, in many cases, in synergy. Synergism means that the AA effect of two or several antioxidant close molecules is higher than the arithmetical sum of the effects of all molecules.

This can be explained by the fact that when the AA molecule prevents the oxidation of the target-compound, its chemical structure changes again to be able to prevent the other target-molecules from recovering the initial structure. Some AA is capable of protecting the molecule effectively, so that other AA can regenerate the AA with changed structure, i.e. they can re-establish the initial form. If there are many AA in the system, in each process there is a molecule that can accomplish the function with maximum efficiency. Therefore, some AA can consolidate the effect of other AA. Synergetic substances, together with AA, play the roles of complexation of the metal ions that can initiate self-oxidation. FR (normal products of cell metabolism) can also be engendered by leukocytes (leukocytes generate FR to destroy phagocytosed "intruders"), from the vascular endothelium (regulation of the contractions of blood vessel smooth muscles), and from hematia. To control the super-production of FR and their action, associated with the presence of pollutant factors from the environment, which also generate FR, the cells develop antioxidant enzymatic systems.

Other substances that are not enzymes are AA: glutathione, vitamins $\mathrm{A}, \mathrm{C}$ and $\mathrm{E}$ etc. The accumulation of an increased amount of FR can be directly related to ageing and to the shortening of the life span. The AA protection mechanism acting against the reactions of the FR is made up of enzymatic and non-enzymatic elements part of which is synthesised only in plants and the body can get them only from foods, while others-first of all enzymes, glucose, uric acid-can also be produced by both the human body and the animal body. Enzymes represent protein molecules. Chemical-biological reactions occurring in the body are catalysed by enzymes. In living bodies, chemical reactions cannot occur without enzymes.

Another characteristic of enzymes is the fact that they do not change during the reaction.

Enzymes are the best known substances of the antioxidant protection system (CAT, GRx, and SOD) and they achieve the alteration of the FR. Enzymes are important components of the protection/defence mechanisms. Enzymes eliminate toxic substances from the body together with bio molecules, mainly hereditary genetic material, and the so-called regulation-repair enzymes that repair the damage of the DNA structure. The existence of living organisms is conditioned by the activity of some resistance and immunity mechanisms capable of protecting their chemical individuality through recognition and differentiation mechanisms of self-substances from non-self-substances [9].

These mechanisms are tolerant to self-molecules, but they activate and react more or less rigorously to remove, neutralise or destroy non self-substances [10]. FR action is an inevitable, continuous process, but when it becomes excessive, it is destructive. When the organism's cells are not protected from the oxidative attack, cell damage can be disastrous. To play a role in the destruction of the FR, antioxidants need: to be present in the proper place and in proper amounts (FR have a short life span); to have a non-specific character to react with radical species; to be distributed widely in the intra-cellular space or to be translocatable; to be synthesised by the cells or to be part of nutrition 
and to be regenerable. AA action is due to the fact that these substances have an increased ability of bonding oxygen, compared to glycerides, PUFA that bond with more difficulty, for instance, a-tocopherol and synthesis substances (propel, octal and dodecyl gallate). Certain chemical substances increase the antioxidant effect of these substances: citric acid and ascorbic acid.

The synergic effect is due to the blocking of metals favouring fat rancidness. Organisms have natural ways of preventing and correcting the occurrence of errors and atypical cell-the fight against FR is carried out with the help of AA who bring the cell back to a neuter condition from a redox point of view. The physiological role of AA is to prevent the destruction of cell components that occur as a result of chemical reactions involving FR. Antioxidant activity depends on that part of the molecule with important electron donor properties. Antioxidant effect results from the activity of capturing FR and the metal chelating property. AA is important because they maintain cell and organism systems health.

\section{References}

1. Giacalone M, Di Sacco F, Traupe I, Topini R, Forfori F, et al. (2011) Antioxidant and neuroprotective properties of blueberry polyphenols: a critical review. Nutr Neurosci 14:119-125.

2. Singh N, Rajini PS (2008) Antioxidant-mediated protective effects of potato peel extract in erythrocytes against oxidative damage. Chem-Biol Interact 173:97-104.
3. Takabe W, Li R, Ai L, Yu F, Berliner JA, et al. (2010) Oxidized Low-Density Lipoprotein-Activated c-Jun $\mathrm{NH}_{2}$-Terminal Kinase Regulates Manganese Superoxide Dismutase Ubiquitination. Implication for Mitochondrial Redox Status and Apoptosis. Arterioscler Thromb Vasc Biol 30:436-441.

4. Valko M, Rhodes CJ, Moncol J, Izakovic M, Mazur M (2006) Free radicals metals and antioxidants in oxidative stress-induced cancer. Chem-Biol Interact 160:1-40.

5. Surapaneni KM, Venkataramana G (2007) Status of lipid peroxidation glutathione, ascorbic acid, vitamin $\mathrm{E}$ and antioxidant enzymes in patients with osteoarthritis. Indian J Med Sci 61:9-14.

6. Milne GL, Seal JR, Havrilla CM, Wijtmans M, Porter NA (2005) Identification and analysis of products formed from phospholipids in the free radical oxidation of human low density lipoproteins. J Lipid Res 46:307-319.

7. Cynshi O, Tamura K, Niki E (2010) Design, synthesis, and action of antiatherogenic antioxidants. Methods Mol Biol 610:91-107.

8. Song MY, Lv N, Kim EK, Kwon KS, Yoo YB, et al. (2009) Antiobesity activity of aqueous extracts of Rhizoma Dioscoreae Tokoronis on high-fat diet-induced obesity in mice. J Med Food 12:304-309.

9. Hathwar SC, Bijinu B, Rai AK, Narayan B (2011) Simultaneous recovery of lipids and proteins by enzymatic hydrolysis of fish industry waste using different commercial proteases. Appl Biochem Biotechnol 164:115-124.

10. Butnariu M, Samfira I (2012) Free Radicals and Oxidative Stress. J Bioequiv Availab 4: iv-vi. doi:10.4172/jbb.10000e13. 